\title{
Bases de datos de guías de práctica clínica
}

\author{
Por Carlos González Guitián y María Sobrido Prieto
}

Resumen: Las guías de práctica clínica son recomendaciones sistemáticas empleadas para la toma de decisiones en la atención sanitaria ante problemas clínicos específicos. De acuerdo con la metodología empleada para su elaboración pueden ser documentos de consenso, opinión de expertos o basados en la evidencia. Tradicionalmente eran entendidas como literatura gris, debido a la difícil localización de las mismas. La aparición de internet ha favorecido sustancialmente su localización. Numerosos organismos oficiales y asociaciones profesionales han posibilitado el acceso a las guías mediante bases de datos y buscadores específicos.

Palabras clave: Guía de práctica clínica, Literatura médica, Bases de datos, Medicina basada en la evidencia.

\section{Title: Databases of clinical practice guidelines}

Abstract: Clinical practice guidelines are systematically developed recommendations to assist in decision-making about appropriate health care for specific clinical circumstances. Depending upon the methodology used, they can be based on consensus, expert opinion, or evidence. Traditionally, these documents were considered as grey literature because of the difficulty in locating them. The arrival of Internet has substantially improved chances for locating them. Numerous institutions and professional associations have facilitated access to them through specialised databases and search engines.

Keywords: Clinical guidelines, Medical literature, Databases, Evidence-based medicine.

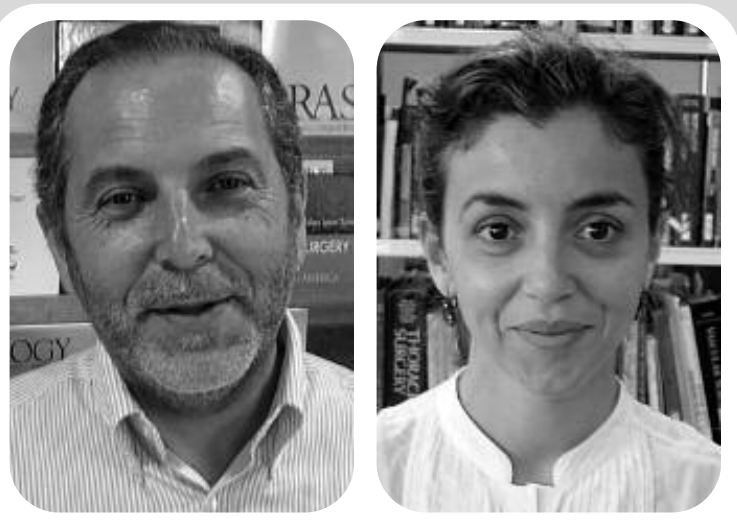

Carlos González Guitián y María Sobrido Prieto

González Guitián, Carlos; Sobrido Prieto, María. «Bases de datos de guías de práctica clínica». En: El profesional de la información, julio-agosto, 2006, v. 15, n. 4, pp. 297-302.

\section{Introducción}

\section{LAS GUÍAS DE PRÁCTICA} CLÍNICA (GPC) son un conjunto de recomendaciones desarrolladas sistemáticamente para informar a profesionales $y$ pacientes sobre las decisiones clínicas. Proporcionan los criterios convencionales y vigentes que expresan las mejores alternativas y orientan las decisiones médicas ${ }^{1,2}$ $y$, por lo tanto, contribuyen a disminuir la falta de homogeneidad ${ }^{3}$.

$\mathrm{Su}$ origen lo encontramos en los años 70, cuando los National Institutes of Health norteamericanos dieron por primera vez respuesta al problema de la variabilidad organizando conferencias de consenso ${ }^{4}$. Con el auge del movimiento de la medicina basada en la evidencia (MBE) en los 90 estas conferencias fueron adquiriendo una organización más formal y configuraron las actuales GPC.
Su importancia ha sido reconocida por gran parte de los profesionales de la salud, pero no existe todavía normalización terminológica al respecto. Así, bajo una misma entidad son entendidas tanto las GPC basadas en la evidencia, conferencia de consenso o basadas en opinión de expertos, pudiendo observarse grandes diferencias en su metodología e incluso en las recomendaciones propuestas de unos a otros documentos ${ }^{4}$. En la literatura anglosajona se pueden encontrar bajo varios términos: guidelines, pathways o guidelines of clinical practice $^{5}$. También existen iniciativas para normalizar su presentación ${ }^{6}$.

Tradicionalmente estos documentos eran considerados como literatura gris, ya que se editaban a través de instituciones o asociaciones profesionales que las distribuían únicamente en el entorno restrin- gido donde debían aplicarse, y en raras ocasiones se publicaban en revistas médicas. Por este motivo su localización era una tarea ardua, bien por no estar indexadas en las bases de datos tradicionales, o porque no contaban con estrategias óptimas de búsqueda bibliográfica ${ }^{6}$. Por ejemplo, en Medline pueden haberse clasificado indistintamente bajo Guidelines, Consensus, Practice guidelines y Consensus development conference. En el caso de otras bases de datos bibliográficas de ámbito internacional, como Embase, es necesario recurrir a la búsqueda por título y/o descriptor. Lo mismo sucede en otros productos biosanitarios españoles como IME, Cuiden, ISOC, etc.?

El concepto de literatura gris para definir este tipo de documentos se ha transformado debido sobre todo a la aparición de internet, que ha propiciado su difusión hasta el 
punto de que su visibilidad es en ocasiones incluso mayor que la de los materiales tradicionales o impresos. Además, y aunque no hay establecido un procedimiento contrastado de búsqueda, numerosos organismos oficiales y asociaciones profesionales tienen una larga tradición en la elaboración de guías de gran calidad y facilitan su acceso por la Red. En otras ocasiones son los centros encargados de recopilar guías a través de bases de datos especializadas. El último instrumento para su localización resulta ciertamente novedoso, ya que se trata de buscadores específicos.

\section{Centros productores y evaluadores de GPC}

Existe una importante multiplicidad de organismos, la mayoría sociedades científicas, pero cuentan con un número reducido de estos documentos. En general todos ellas ofrecen un listado alfabético de las publicaciones disponibles en texto completo (la poca cantidad no justifica un motor de búsqueda).

\section{Scottish Intercollegiate Gui- delines Network (SIGN)}

Destaca por encima del resto. Desde 1993 es una red integrada por colegios de todas las especialidades médicas así como otras profesiones afines a la medicina de Escocia. También participan asociaciones de pacientes, encargados de servicios médicos, servicios sociales e investigadores. Desde el punto de vista metodológico, estas GPC serían las más completas, ya que se elaboran exclusivamente a partir de revisiones sistemáticas de la litera-

\begin{tabular}{|c|}
\hline Browse \\
\hline $\begin{array}{l}\text { क Disease / Condition } \\
\text { क Treatment/Intervention } \\
\text { क Measures / Tools } \\
\text { क Orqanization } \\
\text { क Guideline Index } \\
\text { क Guidelines In Progress } \\
\text { क Guideline Archive }\end{array}$ \\
\hline
\end{tabular}

\begin{tabular}{|c|} 
Compare \\
$\rightarrow \frac{\text { View Mr Collection }}{\text { Guideline Syntheses }}$ \\
\hline
\end{tabular}

National Guidelines Clearinghouse

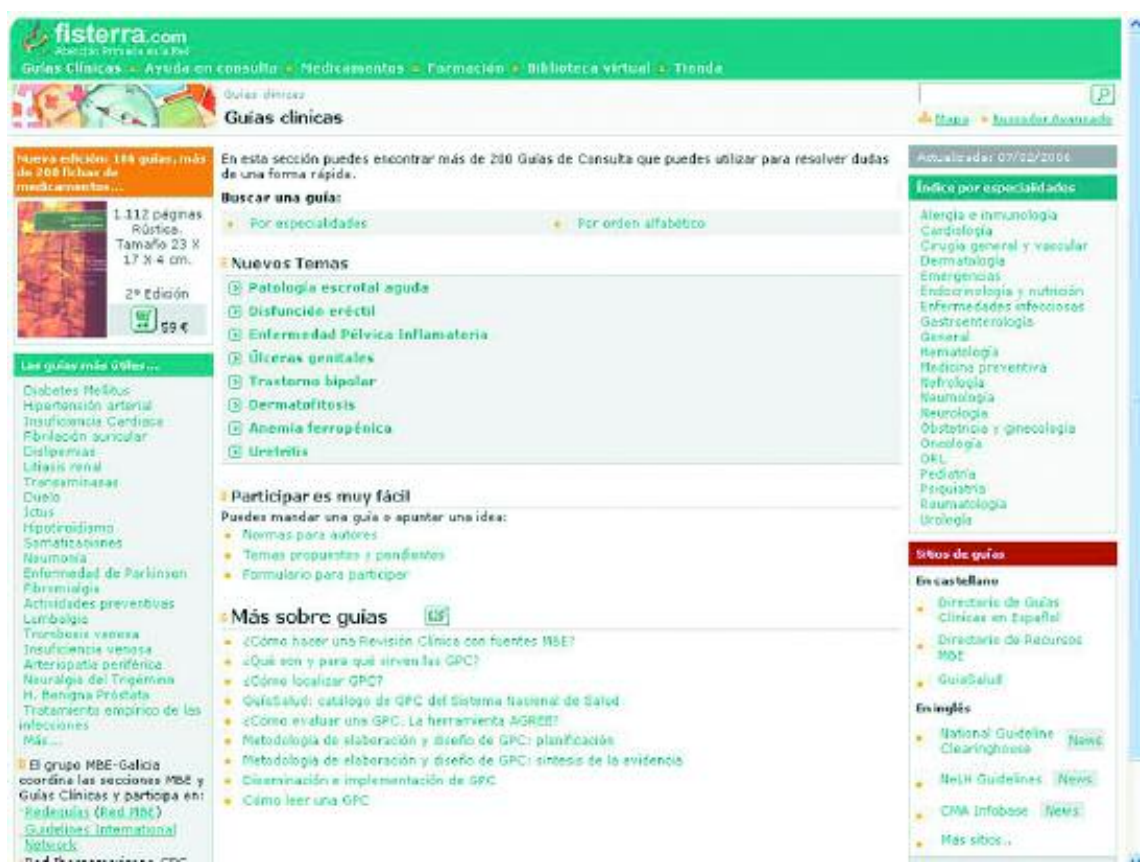

Fisterra

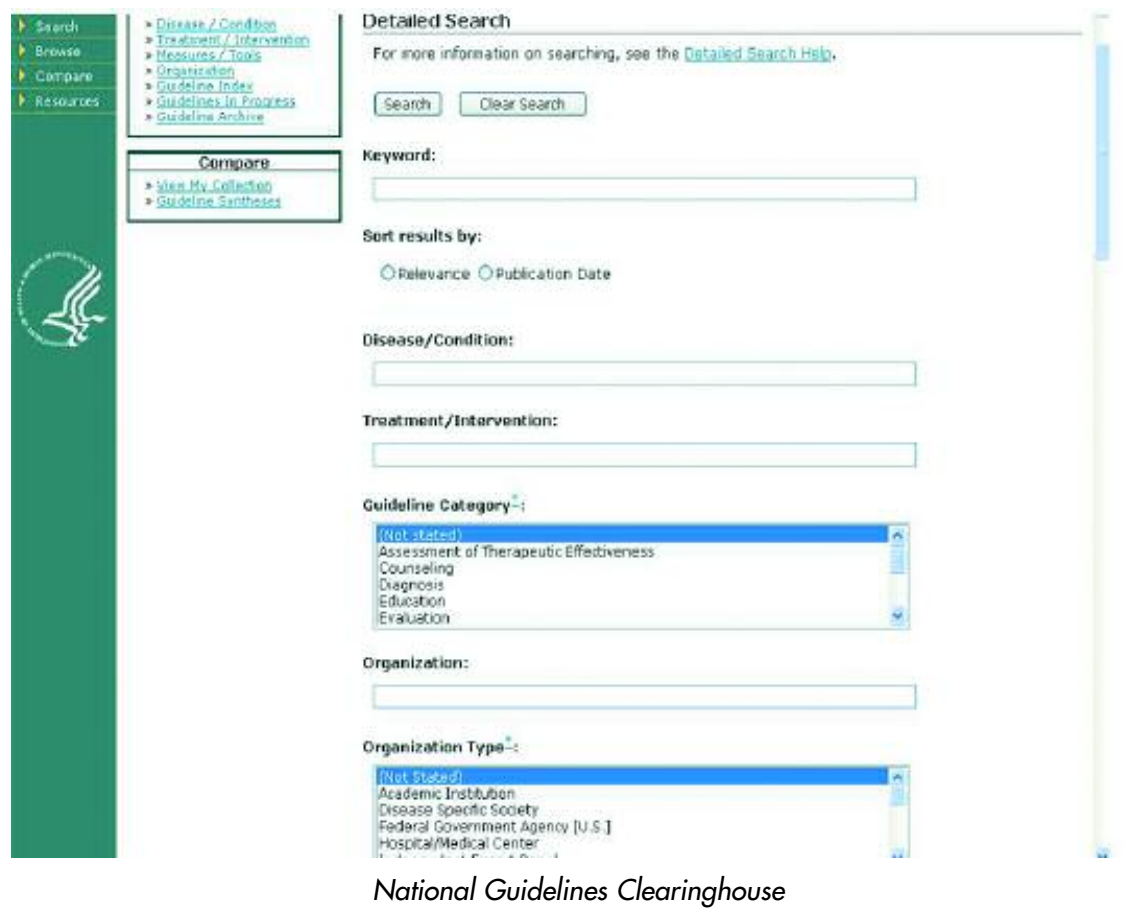

tura, emplean grupos multidisciplinares y exigen que se especifique el grado de evidencia científica que apoya cada recomendación. Las GPC están clasificadas por categorías terapéuticas y disponen de capítulos dirigidos a los diferentes usuarios de las mismas.

\section{New Zealand Guidelines Group (NZGG)}

Se trata este de un grupo de expertos en el desarrollo e implantación de guías con el apoyo del $\mathrm{Na}$ tional Health Committee (Nueva
Zelanda). Supervisa la formación de profesionales en la realización de guías. Dispone de un buscador a través del cual se pueden localizar las guías por especialidad y metodología de elaboración, e incluye un listado de las de producción propia. Están clasificadas por categoría terapéutica, pero además puede usarse un motor de búsqueda con dos sistemas: básico y avanzado (por organización productora, palabras clave, categoría, fecha de publicación y estatus de la publicación). Asumen el Agree (Appraisal 


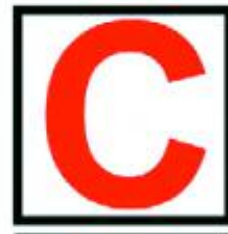

Desarrollo de Indicadores

\section{Líneas de}
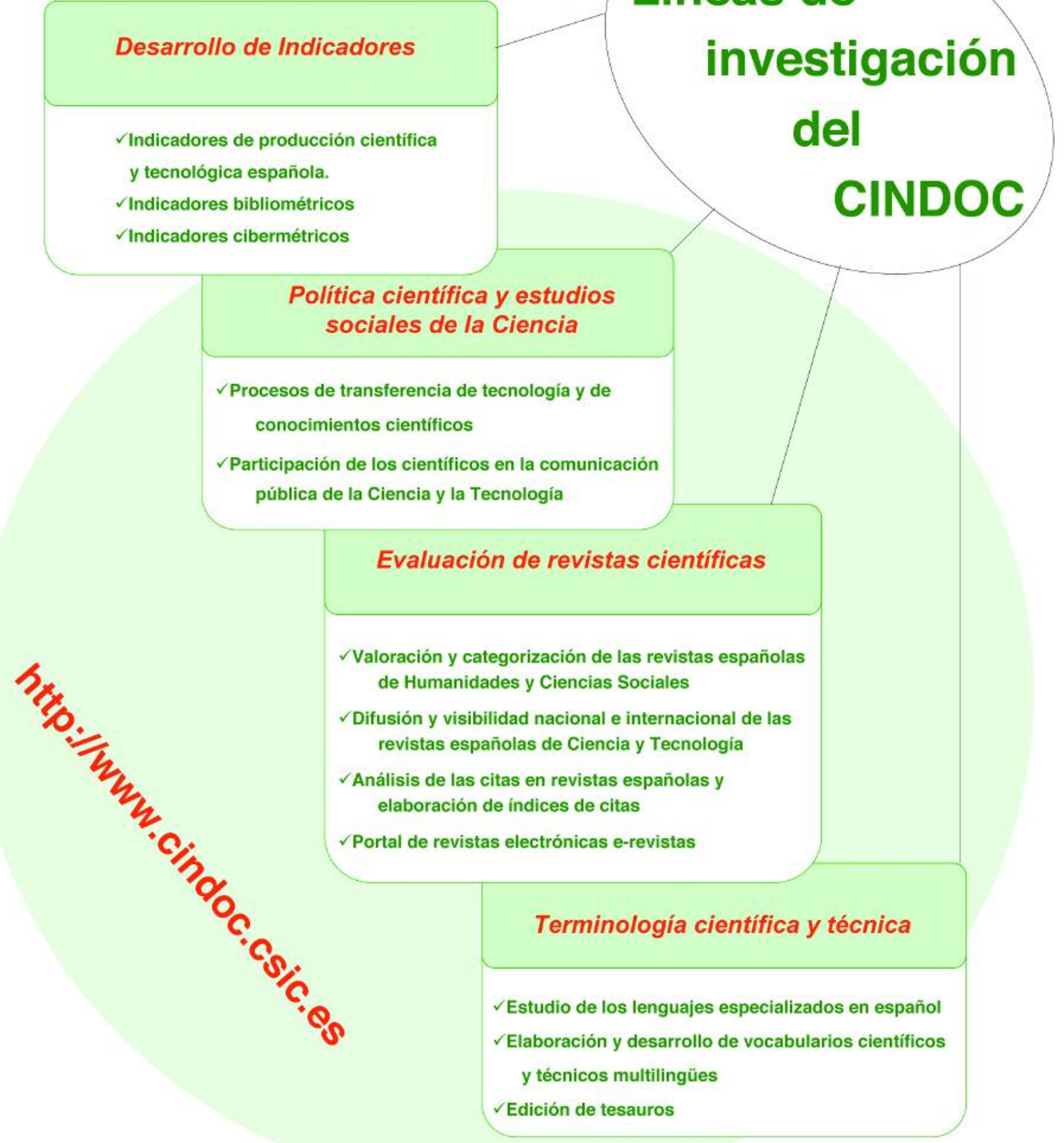


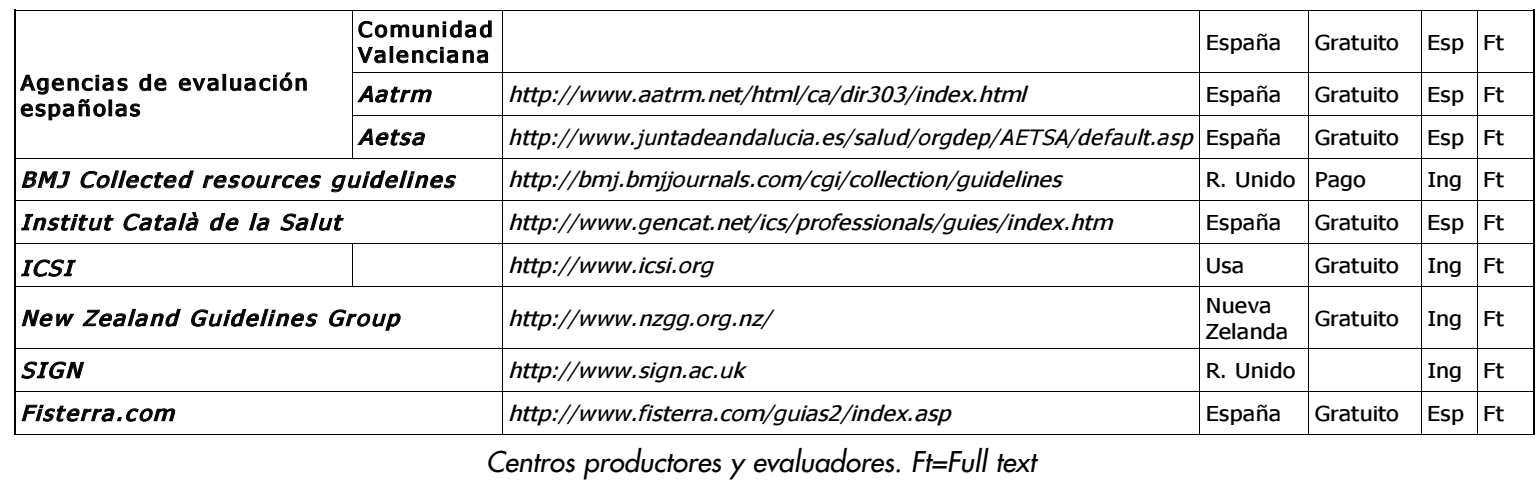

of guidelines research and evaluation $)^{10}$ como instrumento de evaluación de la calidad de las GPC (tanto de la información como de algunos aspectos de las recomendaciones, así como ofrece una valoración de la validez de una guía, es decir, la probabilidad de que logre los resultados esperados ${ }^{8}$ ).

\section{Institute for Clinical System Improvement (ICSI)}

Es un proyecto independiente desarrollado por 6 organizaciones gubernamentales americanas que ofrece servicios de asesoramiento para la mejora de la calidad a los grupos afiliados. Junto con $N Z G G$ y SIGN es uno de los mayores productores de guías de actuación clínica desde una perspectiva de la MBE, orientadas a la atención primaria. Su característica más importante es la cuidadosa elaboración de los diagramas de flujo y algoritmos de decisiones clínicas. Cuentan con herramientas y productos para pacientes, estudios de impacto de implementación y protocolos de cuidados en salud.

En España cabe destacar el trabajo llevado a cabo por las agencias de evaluación catalana y andaluza. Sobresale la Agencia Catalana de Evaluación de Tecnologías Sanitarias (primera española) que elabora recursos metodológicos y un programa de guías oncológicas, Oncoguías, accesibles en su web. Conviene subrayar los casos de algunas consejerías de salud como la de la Comunitat Valenciana, el Institut Català de la Salud y la Consejería de Salud de Andalucía, que también elaboran guías clínicas, aunque desde un concepto más amplio.

Junto a las iniciativas institucionales han surgido en los últimos años en España proyectos privados de interés. Tal es el caso de Fisterra, que incluye más de 300 guías así como documentación sobre la metodología para su comprensión y/o elaboración.

\section{Centros de almacenamiento o clearinghouses}

No elaboran GPC, sino que sólo las localizan, las evalúan y las difunden.

\section{National Guidelines Clea- ringhouse (EUA)}

Base de datos internacional de GPC y documentos relacionados elaborada por la Agency for Health Research and Quality (AHRQ) en colaboración con la American $M e$ dical Association (AMA) y la America's Health Insurance Plans (AHIP).

Aunque apareció hace sólo unos años se ha convertido en uno de los mejores referentes para buscar GPC en internet. La capacidad de búsqueda está muy mejorada y permite dos posibilidades: sencilla y detallada (por categoría, especialidad, metodología de elaboración, etc.). Adicionalmente una tercera posibilidad Frequent searches proporciona una relación de las guías más consultadas.

Asimismo es posible utilizar diferentes opciones en la consulta:
-Disease/condition: con descriptores MeSH (Medical subject headings) o por término libre.

-Treatment/intervention: medicamentos, resultados de intervenciones, diagnósticos, análisis, terapéutica, y salud mental.

-Organization: centro que las ha elaborado y/o recopilado.

-Compare: cotejar guías entre sí para comprobar la metodología de elaboración.

\section{CMA Infobase}

Elaborada por la Canadian Medical Association, reúne guías canadienses de organizaciones profesionales, paneles de expertos, etc. Ofrece acceso al texto completo en inglés y en ocasiones en francés, con búsqueda básica y avanzada (materia, tipo de publicación, etc.). Usan Agree.

\section{EBM Guidelines}

Guías breves realizadas por la Finnish Medical Society (Duodecim) a partir de documentos MBE. La mayor parte se centra en dermatología y oftalmología así como audios de cardiología y neumología. $\mathrm{Su}$ acceso es de pago.

\section{NeLH Guidelines Finder (NeLH)}

También conocida como la Biblioteca Virtual del Servicio de Salud Inglés, facilita el acceso a un importante volumen de recursos electrónicos. Se trata de guías elaboradas en el Reino Unido provenientes de los siguientes instituciones: National Institute for Clinical 
Excellence (NICE); Medendium Group Publishing E-guidelines (acceso mediante suscripción), Prodigy Guidance, Public Health Laboratory Service, Scottish Intercollegiate Network $(S I G N)$, y editoriales como el $B M J$. Permite buscar por especialidad y centro productor.

El Ministerio de Sanidad español ha creado la base de datos específica Guiasalud", un catálogo de GPC elaboradas o adaptadas en España, en cualquiera de las lenguas oficiales del estado. Proporciona información acerca de la elaboración, difusión, utilización y evaluación de GPC.

Para que sean incluidas en el catálogo deben cumplir 6 criterios consensuados por todas las comunidades autónomas y avalados por un comité científico. El propósito es asegurar unos mínimos de calidad y transparencia científica sin que sean herramientas de trabajo de obligado cumplimiento.

Se pueden buscar por especialidad, palabras del título y objetivo general de la GPC.

En Alemania tenemos la German Guideline Clearinghouse, en Francia el Cismef (Catalogue et Index des Sites Médicaux Francophones) a través del Répertoire des Recommandations de Bonne Pratique $\&$ des Conférences de Consensus Francophones, y en Brasil Diretrizes Médicas, auspiciadas por la Associação Médica Brasileira y el Conselho Federal de Medicina.

\section{Otros lugares donde localizar GPC}

Como se señaló al principio, son muchas las instituciones y sociedades científicas que ponen sus GPC en internet. Aunque algunas están recogidas en los centros ya reseñados, en ocasiones es necesario acudir a las sedes para obtener los textos completos. Para localizarlas debemos recurrir a herramientas de búsqueda.

-TripDatabase es un motor de búsqueda específicamente diseñado para localizar documentos médicos de alto valor añadido disponibles en la Red, entre los que se encuentran los principales centros productores de GPC. Busca información en unas 75 sedes de alta calidad relacionadas con la MBE. Comenzó en 1997 como un pequeño buscador y en la actualidad realiza búsquedas en sitios seleccionados por su calidad: revistas con revisión por pares, libros electrónicos, etc., así como GPC. Es gratuito hasta 5 búsquedas semanales; a otra versión más completa, TripDatabasePlus, se accede mediante suscripción.

- Pubgle combina la calidad de indexación de Pubmed con la potencia de búsqueda de Google, mediante esta estrategia de búsqueda:

Guideline[ptyp] OR Practi$c e+$ Guideline [ptyp] OR «Guidelines»[MeSH Terms] OR («health planning guidelines»[MeSH Terms] OR health-planning-guidelines[Text Word]) OR Consen- sus + Development + Conference $[\mathrm{Pu}$ blication Type]

A todo ello se añade el término introducido en el formulario de búsqueda. Los resultados son los mismos que los obtenidos en $P u b$ $m e d$, a cada artículo se añaden dos enlaces: «Búsqueda en internet»y «Enlace» (o el logo de la revista). El primero realiza la búsqueda en Google del título del artículo, el segundo nos lleva al enlace externo del artículo tal como aparece en Pubmed.

Pubmed si bien presenta una serie de dificultades (tal y como se señaló al principio de este trabajo), es una herramienta más para la localización de este tipo de documentos mediante la opción limits.

Puede limitarse la búsqueda de un término a un campo específico. Tal es el caso del tipo de publicación o tipo de estudio publicado (ej. Clinical trials or Review articles). El menú desplegado contiene un listado: Clinical trial; Editorial; Letter; Meta-analysis; Practice guideline; Randomized controlled trial; Review.

\section{Conclusión}

La década de los años 90 ha sido la de la publicación y difusión de las GPC. Las posibilidades que internet ha abierto son enormes, hasta el punto de replantearnos si este tipo de documentos sigue siendo «literatura gris». Tanto el potencial de la Red para la localización de ciertos recursos, como la crea-

\begin{tabular}{|l|l|l|l|l|}
\hline Cma Infobase & http://mdm.ca/cpgsnew/cpgs/index.asp & Canadá & Asociación & Inglés/ Francés \\
\hline $\begin{array}{l}\text { National Guideline } \\
\text { Clearinghouse }\end{array}$ & http://wwww.guideline.gov & EUA & Institución & Inglés \\
\hline NeLH Guidelines Finder & http://libraries.nelh.nhs.uk/guidelinesFinder & R. Unido & Institución & Inglés \\
\hline NICE-Clinical Guidelines & http://wwww.nice.org.uk & R. Unido & Institución & Inglés \\
\hline Guiasalud & http://wwww.guiasalud.es & España & Institución & Español \\
\hline EBM Guidelines & http://wwww.ebm-guidelines.com & Finlandia & Asociación & Inglés \\
\hline $\begin{array}{l}\text { German Guideline } \\
\text { Clearinghouse }\end{array}$ & $\begin{array}{l}\text { http://wwww.leitlinien.de/clearingverfahren/ } \\
\text { english/OOindex/view }\end{array}$ & Alemania & Asociación & Alemán \\
\hline Diretrizes médicas & http://wwww.projetodiretrizes.org.br & Brasil & Asociación & Portugués \\
\hline CisMEF & $\begin{array}{l}\text { http://wwww.chu-rouen.fr/cismef/ } \\
\text { cismef.html }\end{array}$ & Francia & Institución & \\
\end{tabular}

Centros de almacenamiento o clearinghouse. Ft=Full text 


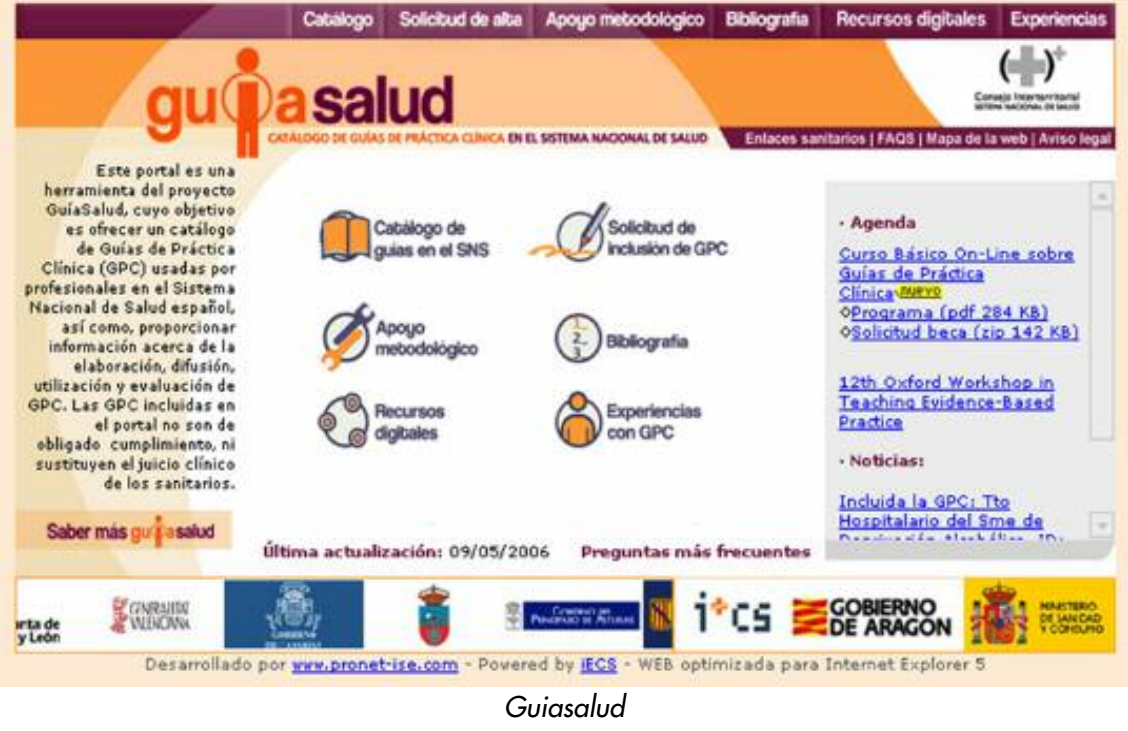

ción de los centros de creación y/o difusión, ha hecho de las GPC documentos de fácil localización y uso frecuente entre la comunidad sanitaria.

La mayoría de los centros creadores/recopiladores (con excepción de la $N G C$ ) son iniciativas nacionales que recogen las guías elaboradas o publicadas en cada país. La $N G C$ tiene la mayor base de datos internacional.

Las posibilidades de búsqueda se encuentran en general bastante desarrolladas; aportan un vocabulario documental controlado (MeSH) en sus guías y ofrecen un pequeño resumen de sus contenidos. La $N G C$ es novedosa en cuanto que permite comparar diferentes documentos.

Además, en la mayoría de los casos los documentos se proveen gratuitamente y a texto completo.

A pesar de todo ello en la actualidad sigue existiendo falta de homogeneidad, tanto en la presentación como en las posibilidades de búsqueda. Asimismo los recursos se han hecho con criterios metodológicos diferentes, lo que obliga a los profesionales de la documentación a un mayor cribado en la búsqueda. Un ejemplo de buen hacer es la National Guidelines Clearinghouse, única iniciativa internacional de todas las mencionadas, que podría llamarse el «Medline de las guías».

Aunque con retraso, en España tenemos Guiasalud, creada por el Sistema Nacional de Salud. De cualquier forma todavía queda mucho por hacer. Es necesario incrementar el número de valores añadidos, tales como resúmenes estructurados, descriptores o la traducción al inglés, bien sea de los resúmenes o los textos completos. Asimismo es necesario definir claramente los criterios metodológicos y establecer el nivel de evidencia de los documentos.

\section{Bibliografía}

1. Clancy, Carolyn M.; Cronin, Kelly. «Evidence-based decision making: global evidence,

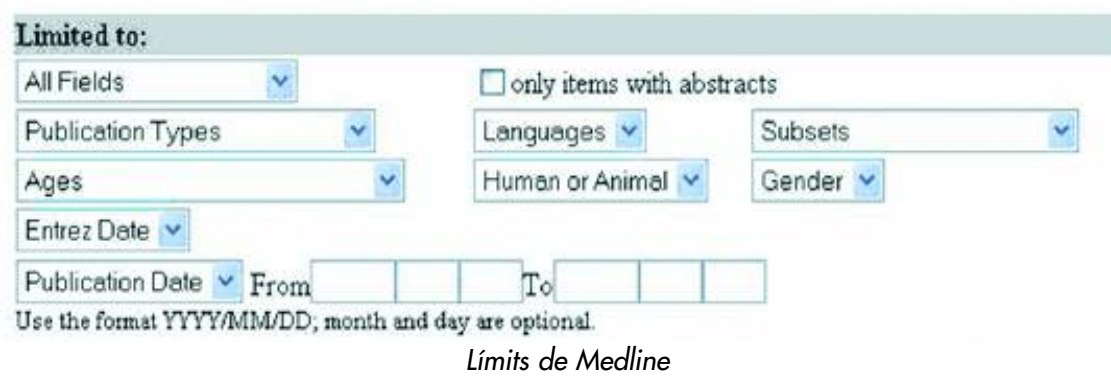

local decisions». En: Health affairs, 2005, enero/febrero, n. 24, pp. 151-162.

2. Louro González, Arturo; Marín León, I. (coord.). Guías de práctica clínica: conceptos básicos sobre su elaboración y utilización. A Coruña: Casitérides, 2006. ISBN 978-84-609-9819-8.

3. Feder, Gene; Eccles, Martin; Grol, Richard; Grifith, Chris; Grimshaw, Jeremy. "Clinical guidelines: using clinical guidelines». En: BMJ, 1999, marzo, v. 318, n. 7.185, pp. 728-30.

4. García Caballero, Manuel. «¿Qué son las GPC? Diferencias con protocolos, algoritmos y vías clínicas». En: García Caballero, M. (coord). Guías de práctica clínica en la asistencia médica. Málaga: Universidad de Málaga. http://webadeptos.uma.es/guia.pdf

5. Field, Marilyn J.; Lohr, Kathleen N. (eds.) Guidelines for clinical practice: from development to use. Washington (DC): Institute of Medicine, National Academy Press, 1992. ISBN 0-309-04589-4.

6. Field, Marilyn J.; Lohr, Kathleen N. (eds.). Clinical practice guidelines: directions for a new agency. Institute of Medicine. Washington (DC): National Academic Press, 1990, p. 58.

7. Peleg, M.; Boxwala, A. A.; Tu, S.; Zeng, Q.; Ogunyemi, O.; Wang, D.; Patel V. L.; Greenes, R. A.; Shortliffe, E. H. «The InterMed approach to sharable computer-interpretable guidelines: a review». En: American Medical Information Association, 2004, Jan.-Feb., v. 11, n. 1, pp. 1-10.

8. López Madurga, Eva; Salcedo Fernández, Flavio; Martín, Juan Ignacio. Guiasalud. $\mathrm{Ca}$ tálogo de GPC en el SNS. Consultado en: 06-03-06.

http://www.fisterra.com/bd/upload/Guiasalud.pdf

9. Martín Muñoz, P.; Ruiz-Canela Cáceres, J.; Guerra de Hoyos, J. A.; Rivas Aguayo, L. Guías de práctica clínica en internet: cómo separar el grano de la paja. En: Revista de pediatría en atención primaria, 2003, abril/junio, v. 5, pp. 73-88.

10. The Agree Collaboration. Agree instrument Spanish version.

http://www.agreecollaboration.org

11. Parada Martínez, Antoni; Aymerich Pons, Marta; Vianney, Joan Maria. «Búsqueda y recuperación de recomendaciones para la práctica clínica en las bases de datos bibliográficas españolas». En: X Jornadas nacionales de información y documentación en ciencias de la salud, 2005, pp. 87-94.

Carlos González Guitián, Departamento de Ciencias de la Salud, Universidade da Coruña. cgongui@canalejo.org

María Sobrido Prieto, Biblioteca
Complexo Hospitalario Universitario
Juan Canalejo.
msobpri@canalejo.org

María Sobrido Prieto, Biblioteca Juan Canalejo. msobpri@canalejo.org 


\section{- organised}

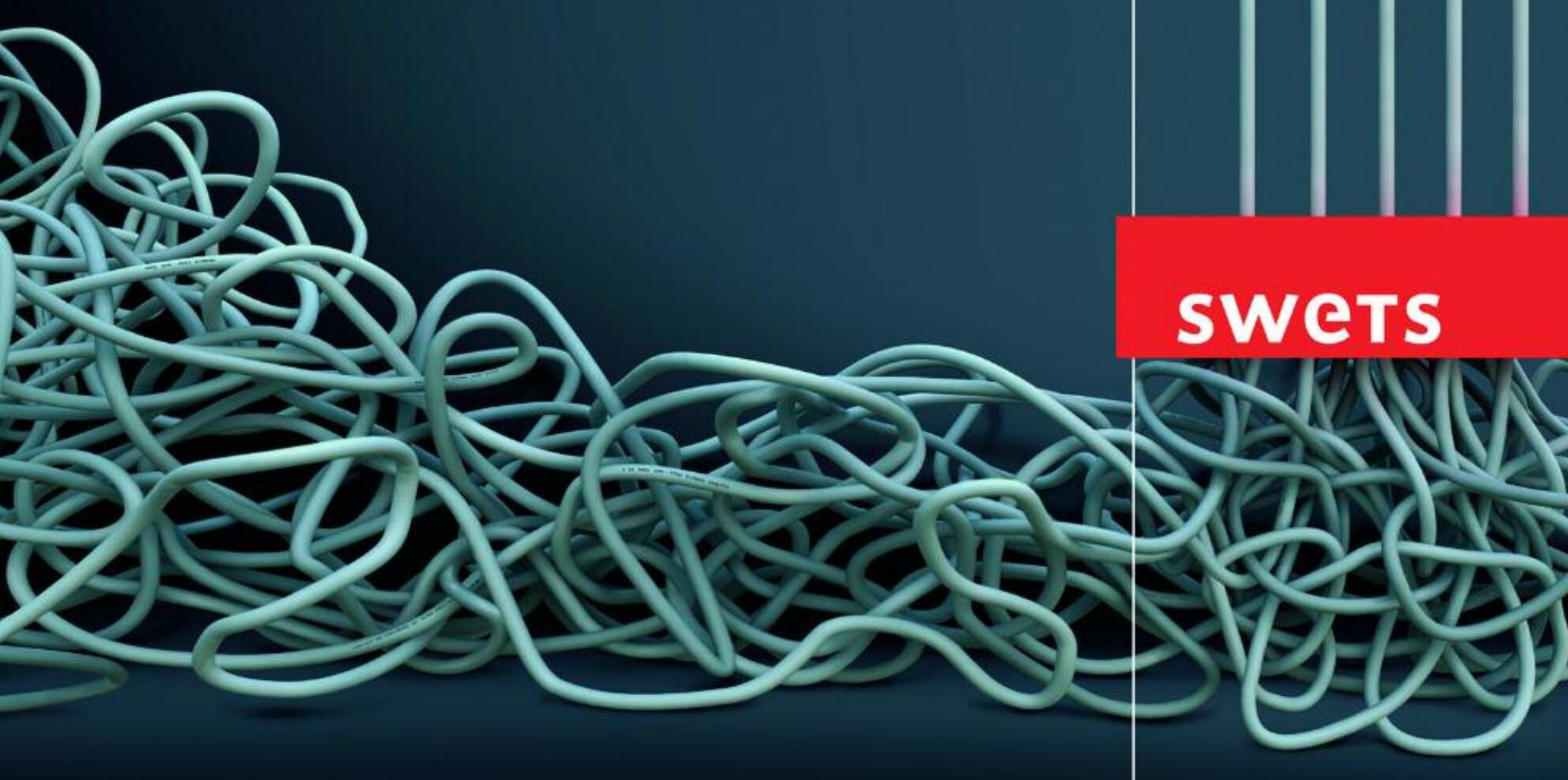

